\title{
Coronectomy may be a way of managing impacted third molars
}

\author{
Abstracted from \\ Long H, Zhou Y, Liao L, Pyakurel U, Wang Y, Lai W. \\ Coronectomy vs. total removal for third molar extraction: a systematic review. \\ J Dent Res 2012; 91: 659-665. \\ Address for correspondence: W. Lai. State Key Laboratory of Oral Diseases, \\ Department of Orthodontics, West China School of Stomatology, Sichuan University, \\ Chengdu, Sichuan 610041, China. E-mail: wenlilai@hotmail.com
}

\section{Question: In patients with impacted third molars does coronectomy when compared to conventional removal result in fewer complications?}

Data sources TPubMed, Embase, Web of Science, Cochrane Central Register of Controlled Trials (CENTRAL) and the grey literature database SIGLE.

Study selection Randomised controlled trials (RCTs) and nonrandomised controlled trials (CCTs) that compared coronectomy with total removal for third molar extractions with high risk of nerve injury were included.

Data extraction and synthesis Data were extracted independently and in duplicate by two reviewers. Risk of bias was assessed according to the Cochrane Reviewers' Handbook. Meta-analysis and sensitivity analysis were performed.

Results Four studies (two RCTs and two CCTs) involving 699 patients and 940 third molars were included. Pooled risk ratios for coronectomy compared with total removal are shown in table 1.

\section{Table 1 Pooled risk ratios coronectomy v total removal}

\begin{tabular}{l|l|l} 
& $\begin{array}{l}\text { Pooled } \\
\text { Risk Ratio }\end{array}$ & $\begin{array}{l}\text { 95\% Confidence } \\
\text { Interval }\end{array}$ \\
\hline Inferior alveolar nerve injury & 0.11 & $0.03-0.36$ \\
\hline Post-operative infection & 1.03 & $0.54-1.98$ \\
\hline Dry socket & 0.55 & $0.28-1.05$ \\
\hline Pain at 1 wk after surgery & 1.14 & $0.57-2.30$
\end{tabular}

Coronectomy was changed to total removal during surgery due to root loosening or mobilisation in $2.3 \%$ to $38.3 \%$ of cases. In $0 \%$ to $4.9 \%$ of cases reoperation was required in the coronectomy group due to persistent pain, root exposure or persistent apical infections. Root migration was only reported in three studies and ranged from $13.2 \%$ to $85.9 \%$.

Conclusions We suggest that coronectomy can protect inferior alveolar nerves in the extraction of third molars with high risk of nerve injury as compared with total removal, and that the risk ratios of postoperative infections were similar between the two surgical modalities.

\section{Commentary}

Coronectomy is a surgical procedure designed to avoid manipulation of the inferior alveolar nerve (IAN), by only removing the crown of an impacted third molar while leaving the root undisturbed.

In this systematic review, the authors searched for studies that compared the post-operative outcome of coronectomy with total removal of the third molar. Two RCTs and two CCTs addressed the inclusion criteria and were included. Four outcome measurements (temporary inferior alveolar nerve injury, post-operative infection, dry socket and pain) were statistically pooled in a meta-analysis. The authors concluded that coronectomy reduces the risk of IAN injury without increasing the risk of post-operative infection compared to total extraction.

The authors substantiate the results through solid statistical analyses. The heterogeneity among studies was tested and the sensitivity analyses and Intention to Treat analyses were performed. A quality assessment according to the Cochrane Reviewers' Handbook was performed and the authors concluded that the included studies had unclear or high risk of bias and were therefore of poor to medium quality. However, the authors failed to describe possible confounding of their results due to the low quality of the included studies. If bias is present in some of the studies, meta-analysis will simply compound the errors, and produce a 'false result that may be interpreted as having more credibility'. The authors failed to describe or discuss possible differences in patient characteristics at baseline of the included studies. For example, smoking and poor oral hygiene are significant risk factors for post-operative infection and alveolitis. None of the included studies reported these parameters, which may bias the results, especially if there was no randomisation. If, hypothetically, there were fewer smokers in the coronectomy group compared to the extraction group, the incidence of post-operative infection and alveolitis would be higher in the extraction group. Another important potential bias is the surgeon's skill and experience. The risk of IAN injury in total third molar removal is reduced when an experienced surgeon performs the surgery. The coronectomy is a delicate procedure; the risk of failure of the procedure or post-operative infection would possibly be higher if the surgeon has less experience. These are important confounding factors, which should be addressed. When the primary analysis is based on all studies, we would suggest incorporating the assessments of risk of bias into measures of the quality of evidence, for example using the GRADE system. This can help to ensure that judgments of risk of bias, as well as other factors affecting the quality of evidence, such 
as imprecision and publication bias, are appropriately taken into consideration when interpreting the results.

The authors did not mention the rate of permanent IAN injury in the total extraction groups. Pooling the data, 43 patients out of 521 third molars, $(8.3 \%)$, were diagnosed with IAN injury in the total extraction group. However, the injury resolved in 35 patients within one month after extraction and eight patients, (1.5\%), were diagnosed with permanent IAN injury (>six months). So there was no significant difference between the coronectomy group and total extraction group in the incidence of permanent IAN injury. Still, preventing permanent IAN injury in $1.5 \%$ of cases is very valuable, but the high risk of bias may weaken this number further. The difference in temporary and permanent IAN injury may influence the decision making process whether or not to perform a coronectomy, especially if uncertainties regarding further migration of the roots in the long term and the effect on the periodontal health of the second molar still exists.

The authors conclude that their results are robust and they suggest that coronectomy could be used in clinical practice. In our opinion, coronectomy is a promising procedure. However, it is highly questionable if the results from this systematic review, with a low power and based on studies with a high risk of bias, should already be incorporated routinely in the clinical practice.

More well designed randomised controlled clinical trials with a low risk of bias and longer follow-up terms are needed, to determine the fate of the root and periodontal health of the adjacent second molar in the long term. Ideally, an evaluation of the costeffectiveness of the procedure and the risk of temporary and permanent IAN injury and its impact on Quality of Life should be addressed.

Hossein Ghaeminia

Department of Oral and Maxillofacial Surgery, Radboud University Medical Centre, Nijmegen, The Netherlands.

Evidence-Based Dentistry (2013) 14, 57-58. doi:10.1038/sj.ebd.6400939 